\title{
Efficiently transforming from values of a function on a sparse grid to basis coefficients
}

\author{
Robert Wodraszka and Tucker Carrington Jr. \\ Chemistry Department, Queen's University, Kingston, Ontario K7L 3N6, Canada
}

Summary. In many contexts it is necessary to determine coefficients of a basis expansion of a function $f\left(x_{1}, \ldots, x_{D}\right)$ from values of the function at points on a sparse grid. Knowing the coefficients, one has an interpolant or a surrogate. For example, such coefficients are used in uncertainty quantification. In this chapter, we present an efficient method for computing the coefficients. It uses basis functions that, like the familiar piecewise linear hierarchical functions, are zero at points in previous levels. They are linear combinations of any, e.g. global, nested basis functions $\varphi_{i_{k}}^{(k)}\left(x_{k}\right)$. Most importantly, the transformation from function values to basis coefficients is done, exploiting the nesting, by evaluating sums sequentially. When the number of functions in level $\ell_{k}$ equals $\ell_{k}$ (i.e. when the level index is increased by one, only one point (function) is added) and the basis function indices satisfy $\|\mathbf{i}-\mathbf{1}\|_{1} \leq b$, the cost of the transformation scales as $\mathscr{O}\left(D\left[\frac{b}{D+1}+1\right] N_{\text {sparse }}\right)$, where $N_{\text {sparse }}$ is the number of points on the sparse grid. We compare the cost of doing the transformation with sequential sums to the cost of other methods in the literature.

\section{Introduction}

Sparse grids are often used to interpolate multi-dimensional functions. [9, 18, 23] An interpolant for a function $f\left(x_{1}, x_{2}, \ldots, x_{D}\right)$ is usually built from a set of basis functions and a set of interpolation points. In this chapter, the basis functions are products of 1-D functions which are denoted $\varphi_{i_{k}}^{(k)}\left(x_{k}\right)$, where $i_{k}=1,2, \ldots, n_{k}$ and $k=1,2, \ldots, D$. $D$ is the number of dimensions and $n_{k}$ is the number of basis functions for dimension $k$. All interpolants in this chapter can be written in the general form

$$
\bar{f}\left(x_{1}, \ldots, x_{D}\right)=\sum_{\mathbf{i}} C_{\mathbf{i}} \prod_{k=1}^{D} \varphi_{i_{k}}^{(k)}\left(x_{k}\right),
$$

where the sum is over a set of $\mathbf{i}=\left(i_{1}, \ldots, i_{D}\right)$ indices. Throughout this chapter: a superscript in brackets indicates a particular dimension; $\varphi_{j_{k}}^{(k)}$ is the $j_{k}$-th basis function for dimension $k$; vectors, matrices, and tensors are in bold type, level indices are script $\ell$; the lower case letter $a$ labels a point; and basis set functions are labelled by $i, j, i^{\prime}, j^{\prime}$. The coefficients $C_{\mathbf{i}}$ are determined so that the interpolant is equal to known function values at the interpolation points, 
$\left(r_{a_{1}}^{(1)}, r_{a_{2}}^{(2)}, \ldots, r_{a_{D}}^{(D)}\right)$. Note that $r_{a_{k}}^{(k)}$ is a point in dimension $k$ and that $a_{k}$ labels a point. We shall assume throughout the chapter that the number of interpolation points is equal to the number of basis functions.

A simple and general interpolation method is obtained by using special 1-D basis functions, $G_{a_{k}}^{(k)}\left(x_{k}\right)$, called Lagrange type functions, that are equal to zero at all points except one, a tensor product basis, and a tensor product grid of interpolation points. In this case, the coefficients $C_{\mathbf{i}}$ are simply the known values of the function at the points. The most common Lagrange type functions are the Lagrange polynomials that span the same polynomial space as the monomial basis $\left\{x_{k}^{0}, x_{k}^{1}, \ldots, x_{k}^{n-1}\right\}$. However, it is possible to make Lagrange type functions that span the space spanned by any $1-\mathrm{D}$ basis, see $[3,21]$,

$$
G_{a_{k}}^{(k)}\left(x_{k}\right)=\sum_{j_{k}=1}^{n_{k}}\left[\left(\mathbf{B}^{(\mathbf{k})}\right)^{-1}\right]_{j_{k}, a_{k}} \varphi_{j_{k}}^{(k)}\left(x_{k}\right), a_{k}=1,2, \ldots, n_{k} .
$$

$n_{k}$ is the number of basis functions for dimension $k$. A Lagrange type function is labelled by the point at which it is centred. In this chapter, points for dimension $k$ are labelled by $a_{k}$ or $a_{k}^{\prime}$. In Eq. (2), $\left(\mathbf{B}^{(\mathbf{k})}\right)_{a_{k}, j_{k}}=\varphi_{j_{k}}^{(k)}\left(r_{a_{k}}^{(k)}\right)$. Making these Lagrange type functions requires inverting a small $n_{k} \times n_{k}$ matrix. Instead of Lagrange type functions, it is also common to use a basis composed of piecewise linear functions (hat functions), called a "nodal" basis. [11] In this nodal basis, the coefficients $C_{\mathbf{i}}$ are also the known values of the function at the points.

Any tensor product basis is afflicted by the curse of dimensionality. A better multidimensional basis can be made by using sparse grid or Smolyak type ideas and 1-D basis functions that are importance-ordered. [11] An importance-ordered 1-D basis, $\varphi_{i_{k}}^{(k)}\left(x_{k}\right)$, is one in which a basis function is more important if its value of $i_{k}$ is smaller. An important basis function is one whose coefficient is large. This is the famous idea of Archimedes. [1] For the purpose of interpolating with the sparse-grid Ansatz, it is best to use ZAPPL functions (see section 2), made from an importance-ordered basis. To interpolate with Smolyak's idea one frequently uses [9]

$$
I(D, b)=\sum_{\|\ell-\mathbf{1}\|_{1} \leq b} \Delta^{\ell_{1}} \otimes \Delta^{\ell_{2}} \ldots \otimes \Delta^{\ell_{D}}
$$

where $\ell_{k}$ labels a level.

$$
\Delta^{\ell_{k}}=U^{\ell_{k}}-U^{\ell_{k}-1}
$$

and $U^{\ell_{k}}$ is a 1-D interpolation rule; $U^{0}=0$. [9] In the restriction on the sum, $\|\boldsymbol{\ell}-\mathbf{1}\|_{1}=$ $\sum_{k=1}^{D}\left|l_{k}-1\right|$, where $l_{k}=1, \ldots, b+1 \forall k=1, \ldots, D$. The importance-ordered basis is divided into levels and for each level there is a corresponding set of points. In level $\ell_{k}$, for coordinate $k$, there are $m_{k}\left(\ell_{k}\right)$ points and basis functions. In this chapter, for simplicity, we shall set $m_{k}\left(\ell_{k}\right)=\ell_{k}$, but the same ideas can be implemented when $m_{k}\left(\ell_{k}\right) \geq \ell_{k}$. [3] Everywhere in this chapter, we shall assume that the sequences of points are nested, i.e., the set of points with $m_{k}\left(\ell_{k}\right)$ points includes all the points in the set with $m_{k}\left(\ell_{k}-1\right)$ In this chapter, the grids included in the sum in Eq. (3) are those that satisfy the condition $\|\boldsymbol{\ell}-\mathbf{1}\|_{1} \leq b$, but other choices are possible. [3] The space spanned by the pruned (restricted) basis is smaller than the space spanned by the tensor product basis. If both the function being interpolated and the basis functions are smooth, the pruning is effective. In general, formulations that obviate the sum over levels are less costly than Eq. (3) which requires a sum over levels. 
The interpolant made from Eq. (3) can be written in terms of Lagrange type functions or in terms of the functions $\varphi_{i_{k}}^{(k)}\left(x_{k}\right)$, from which the Lagrange type functions are made. If written in terms of $\varphi_{i_{k}}^{(k)}\left(x_{k}\right)$, the interpolant is

$$
[I(D, b) f]\left(x_{1}, \ldots, x_{D}\right)=\sum_{\|\mathbf{i}-\mathbf{1}\|_{1} \leq b} C_{i_{1}, i_{2}, \ldots, i_{D}} \varphi_{i_{1}}^{(1)}\left(x_{1}\right) \varphi_{i_{2}}^{(2)}\left(x_{2}\right) \ldots \varphi_{i_{D}}^{(D)}\left(x_{D}\right)
$$

Re-writing the sum over levels as a sum over basis indices is only this simple if $m_{k}\left(\ell_{k}\right)=\ell_{k}$. When $m_{k}\left(\ell_{k}\right) \neq \ell_{k}$, the restriction on the basis indices is not the same as the restriction on the levels. [3, 6] By equating Eq. (5) and the Lagrange type function form of the interpolant, one obtains an expression for $C_{i_{1}, i_{2}, \ldots, i_{D}}$ in terms of values of the function on the sparse grid. [3] In Sec. 3, we present simpler ideas for obtaining $C_{i_{1}, i_{2}, \ldots, i_{D}}$. They work only if the sets of points are nested.

It is common to use a basis of piecewise linear functions, divided into levels, that are defined so that the space spanned by the functions in the first $\ell$ levels is the same as the space spanned by the $\ell$-th nodal basis. [11] These functions are called "hierarchical". They are importance-ordered. In addition, they have the property that functions in level $\ell$ are equal to zero at points in levels $1,2, \ldots, \ell-1$. We call this the zero-at-points-in-previous-levels (ZAPPL) property. When basis functions with the ZAPPL property are used, it is not necessary to sum over levels to determine an interpolant. [16, 11, 3] In [24], the ZAPPL property is called the fundamental property. Functions that satisfy the ZAPPL property are also called incremental hierarchical functions. However, in the sparse grid literature they are very often piecewise linear. [11] Ref. [16] is an important exception to this rule. Using the ideas of section 2, it is straightforward to make ZAPPL functions from any global 1-D basis functions. The efficiency of the approach of Sec. 3 relies on using basis functions with the ZAPPL property.

\section{1-D ZAPPL basis functions}

ZAPPL functions can be made from any 1-D basis set which is divided into levels and nested sets of points associated with the levels. A general recipe for making ZAPPL functions is given in Refs. [3, 24]. In those papers the ZAPPL functions are called hierarchical. In this chapter, in level 1 , we have $\varphi_{1}^{(k)}$, in level 2 we have $\left(\varphi_{1}^{(k)}, \varphi_{2}^{(k)}\right)$, in level 3 we have $\left(\varphi_{1}^{(k)}, \varphi_{2}^{(k)}, \varphi_{3}^{(k)}\right)$, etc. Correspondingly, in level 1 , we have the point $r_{1}^{(k)}$, in level 2 we have the points $\left(r_{1}^{(k)}, r_{2}^{(k)}\right)$, in level 3 we have $\left(r_{1}^{(k)}, r_{2}^{(k)}, r_{3}^{(k)}\right)$, etc. The ZAPPL functions are:

$$
\tilde{\varphi}_{i_{k}}^{(k)}\left(x_{k}\right)=\sum_{j_{k}=1}^{i_{k}} \tilde{A}_{i_{k}, j_{k}}^{(k)} \varphi_{j_{k}}^{(k)}\left(x_{k}\right),
$$

where $\tilde{A}_{i_{k}, j_{k}}^{(k)}$ is chosen so that $\tilde{\varphi}_{i_{k}}^{(k)}\left(r_{a_{k}}^{(k)}\right)=0 \quad \forall a_{k}<i_{k} \quad$ and $\quad \tilde{A}_{i_{k}, i_{k}}^{(k)}=1$. Note that $\tilde{\varphi}_{i_{k}}^{(k)}\left(x_{k}\right)$ depend on the interpolation points and $\varphi_{i_{k}}^{(k)}\left(x_{k}\right)$ do not. The 1-D points must be chosen so that $\tilde{\mathbf{B}}^{(k)}$ is not singular, or near-singular, where $\tilde{B}_{a_{k}, i_{k}}^{(k)}=\tilde{\varphi}_{i_{k}}^{(k)}\left(r_{a_{k}}^{(k)}\right)$. In the rest of this chapter we, for simplicity, omit tildes. $B_{a_{k}, i_{k}}^{(k)}$ always means $\tilde{B}_{a_{k}, i_{k}}^{(k)}$ and $\varphi_{i_{k}}$ always means $\tilde{\varphi}_{i_{k}}$.

The ZAPPL functions defined in Eq. (6) have the advantageous ZAPPL property, but they may be smooth and are not the common piecewise linear hierarchical functions. [11] 
To interpolate smooth functions it is often better to use smooth basis functions. Eq. (6) can be used to make ZAPPL functions from any importance-ordered basis. For example, a set of importance-ordered B splines could be used. [24] The prescription of Eq. (6) can be used regardless of the choice of $m_{k}\left(\ell_{k}\right)$, the number of functions in level $\ell_{k}$. In many cases, choosing $m_{k}\left(\ell_{k}\right)$ so that it does not increase exponentially with $\ell_{k}$ reduces the cost of calculations. Of course, it must be possible to choose nested sets of points with $m_{k}\left(\ell_{k}\right)$ points in level $\ell_{k}$. In this chapter, our cost estimates are computed using $m_{k}\left(\ell_{k}\right)=\ell_{k}$, which means that when $\ell_{k}$ is increased by one, we must add a single new point. One way to do this is to use Leja points. $[19,22,8]$

\section{Transforming from function values to basis coefficients}

In this section, we present our efficient scheme for transforming a vector whose elements are values of a function at points on the sparse grid to a vector whose elements are the coefficients of a ZAPPL basis expansion of the function. Everything in this section is valid for any choice of the $\varphi_{i_{k}}^{(k)}\left(x_{k}\right)$ functions and any choice of the (nested) points. We compare our scheme to other transformation methods in the literature. [24, 15, 13] The method of this section was presented at the Sparse Grids and Applications conference in Munich. After the conference, David Holzmueller showed that the ideas can be formulated in terms of LU decompositions. [17] Note that if one wishes basis expansion coefficients in a (nested) basis that is not a ZAPPL basis, one can use the method of this section to transform from the grid to the ZAPPL coefficients and then efficiently (evaluating sums sequentially) transform from the ZAPPL coefficients to the coefficients in the desired basis, see Eq. (41) in Ref. [3]

\subsection{It appears one needs to invert B}

Let $f: \mathbb{R}^{D} \rightarrow \mathbb{R}$ be a multivariate function. Its Smolyak interpolant can be written as in Eq. (5). Eq. (5) is similar to the generalised polynomial chaos expansion (GPCE) employed when solving stochastic differential equations. $[15,27]$ Note, however, that our basis functions are not necessarily (weighted) polynomials; they can be chosen to reduce the size of the basis required for the interpolation. The goal is to obtain the expansion coefficients $C_{\mathbf{i}}$ given the values of the function $f$ at the sparse grid points, i.e, $f\left(r_{a_{1}}^{(1)}, \ldots, r_{a_{D}}^{(D)}\right)$. Explicit equations for the matrix-vector product required to compute $C_{\mathbf{i}}$, Eq. (19), and for the cost, Eq. (25), are simple if the basis function indices in Eq. (5) are restricted by $\|\mathbf{i}-\mathbf{1}\|_{1} \leq b$ and the grid indices are restricted by $\|\mathbf{a}-\mathbf{1}\|_{1} \leq b$. Both these restrictions are inherited from the level restriction in Eq. (3).

The most straightforward approach for obtaining $C_{\mathbf{i}}$ from Eq. (1) is to solve a system of linear equations

$$
\begin{aligned}
\bar{f}\left(\mathbf{r}_{\mathbf{a}}\right) & =f\left(\mathbf{r}_{\mathbf{a}}\right)=\sum_{\|\mathbf{i}-\mathbf{1}\|_{1} \leq b} C_{\mathbf{i}} \prod_{k=1}^{D} \varphi_{i_{k}}^{(k)}\left(r_{a_{k}}^{(k)}\right) \quad \forall\|\mathbf{a}-\mathbf{1}\|_{1} \leq b, \\
\Longleftrightarrow C_{\mathbf{i}} & =\sum_{\|\mathbf{a}-\mathbf{1}\|_{1} \leq b}\left[\mathbf{B}^{-1}\right]_{\mathbf{i}, \mathbf{a}} f\left(\mathbf{r}_{\mathbf{a}}\right) \quad \forall\|\mathbf{i}-\mathbf{1}\|_{1} \leq b .
\end{aligned}
$$

By solving Eq. (8), one obtains all of the coefficients $C_{\mathbf{i}}$ from one calculation. The elements of the matrix $\mathbf{B}$ are values of the basis functions at the sparse grid points, i.e., 


$$
B \begin{gathered}
B a_{1}, \ldots, a_{D} \\
i_{1}, \ldots, i_{D}
\end{gathered}=\prod_{k=1}^{D} \varphi_{i_{k}}^{(k)}\left(r_{a_{k}}^{(k)}\right) \quad \forall\|\mathbf{i}-\mathbf{1}\|_{1} \leq b \text { and }\|\mathbf{a}-\mathbf{1}\|_{1} \leq b .
$$

With the chosen restrictions of the indices, the number of sparse grid points (and product basis functions) is

$$
N_{\text {sparse }}=\left(\begin{array}{c}
D+b \\
D
\end{array}\right)
$$

In general, to solve the linear system of equations, $\mathscr{O}\left(N_{\text {sparse }}^{3}\right)$ floating point operations are required. Directly solving the linear equations in Eq. (8) or inverting B will therefore, especially for high-dimensional problems, require considerable computer time and computer memory. Smolyak interpolation thus has the advantage that $N_{\text {sparse }} \ll(b+1)^{D}$, but the disadvantage that it is not simple to determine $C_{\mathbf{i}}$.

If both the basis and the point set are tensor products, i.e., the restrictions imposed on the indices are $\|\mathbf{i}-\mathbf{1}\|_{\infty} \leq b$ and $\|\mathbf{a}-\mathbf{1}\|_{\infty} \leq b$, then the entire set of coefficients $C_{\mathbf{i}}$ can be easily found because one can exploit the fact that

$$
\mathbf{B}=\mathbf{B}^{(\mathrm{kron})}=\bigotimes_{k=1}^{D} \mathbf{B}^{(k)}
$$

a Kronecker product of small $(b+1) \times(b+1)$ matrices $B_{a_{k}, i_{k}}^{(k)}=\varphi_{i_{k}}^{(k)}\left(r_{a_{k}}^{(k)}\right)$. Hence,

$$
\mathbf{B}^{-1}=\left[\mathbf{B}^{(\mathrm{kron})}\right]^{-1}=\bigotimes_{k=1}^{D}\left[\mathbf{B}^{(k)}\right]^{-1}
$$

This has two advantages. First, it is not necessary to invert a large matrix and second, it is possible to evaluate the sums in Eq. (8) sequentially,

$$
C_{\mathbf{i}}=\sum_{a_{D}=1}^{b+1}\left(\left[\mathbf{B}^{(D)}\right]^{-1}\right)_{i_{D}, a_{D}} \ldots \sum_{a_{2}=1}^{b+1}\left(\left[\mathbf{B}^{(2)}\right]^{-1}\right)_{i_{2}, a_{2}} \sum_{a_{1}=1}^{b+1}\left(\left[\mathbf{B}^{(1)}\right]^{-1}\right)_{i_{1}, a_{1}} f\left(\mathbf{r}_{\mathbf{a}}\right),
$$

as is frequently done in chemical physics to transform between a grid and a basis. [20] Not exploiting the Kronecker product structure of Eq. (11) and solving the linear system directly would require $\mathscr{O}\left((b+1)^{3 D}\right)=\mathscr{O}\left(N_{\text {full }}^{3}\right)$ operations, whereas the sequential summation approach of Eq. (13) requires only

$$
\mathscr{O}\left(D(b+1)^{D+1}\right)=\mathscr{O}\left(D(b+1) N_{\text {full }}\right)
$$

operations, where $N_{\text {full }}=(b+1)^{D}$. Each sum in Eq. (13) can be thought of as calculating $(b+1)^{D-1}$ MVPs for a matrix of size $(b+1)$. As each of these MVPs requires $(b+1)^{2}$ multiplications and there are $(b+1)^{D-1}$ of them, the total cost scales as $(b+1)^{D+1}$. Eq. (14) is the cost of computing the entire set of $C_{\mathbf{i}}$ coefficients.

Although $\mathbf{B}$ in Eq. (8) is not a tensor product, we show in the next subsection that the sparse grid to basis transformation can nevertheless be done sequentially, when ZAPPL basis functions are employed. The numerical cost then scales as

$$
\mathscr{O}\left(D\left[\frac{b}{D+1}+1\right] N_{\text {sparse }}\right) \text {. }
$$


Again, this is the cost of computing the entire set of $C_{\mathbf{i}}$ coefficients. Because in both cases sums are evaluated sequentially, Eq. (15) and the right side of Eq. (14) have the same structure and there is a factor of $D$ in both, but in Eq. (15), $N_{\text {full }}$ is replaced with $N_{\text {sparse }}$ and $(b+1)$ is replaced with $\frac{b}{D+1}+1$.

\subsection{ZAPPL functions and sequential summation obviate the need to invert $B$}

We begin by sorting the products of ZAPPL functions that form the tensor product basis into two groups: the $N_{\text {sparse }}$ functions that are included in the sparse basis go into a group labelled "retained"; and the $N_{\text {full }}-N_{\text {sparse }}$ functions that are excluded from the sparse basis go into a group labelled "discarded". Let $\mathbf{C} \in \mathbb{R}^{N_{\text {full }} \times N_{\text {sparse }}}$ be a chopping matrix which is an $N_{\text {full }} \times N_{\text {full }}$ identity matrix from which the columns for the excluded functions have been deleted. In terms of $\mathbf{C}, \mathbf{B}$ can be written as

$$
\begin{aligned}
\mathbf{B} & =\mathbf{C}^{T} \mathbf{B}^{(\text {kron })} \mathbf{C} \\
\Longleftrightarrow \mathbf{B}^{-1} & =\left[\mathbf{C}^{T} \mathbf{B}^{(\text {kron })} \mathbf{C}\right]^{-1}
\end{aligned}
$$

It is far from obvious that one can calculate elements of the matrix on the right side of Eq. (17) by inverting small matrices for each coordinate and then do the sums in an equation like Eq. (8) sequentially so that one obtains an equation similar to Eq. (13). Both are necessary, if one is to find an inexpensive method for computing $C_{\mathbf{i}}$ on the LHS of Eq. (8). However, if excluded functions are zero at retained points then

$$
\left[\mathbf{C}^{T} \mathbf{B}^{(\mathrm{kron})} \mathbf{C}\right]^{-1}=\mathbf{C}^{T}\left[\mathbf{B}^{(\mathrm{kron})}\right]^{-1} \mathbf{C}
$$

In words, we can interchange the operations of chopping and inverting. The matrix in the matrix-vector product in Eq. (8) is the inverse of the retained block, but because the inverse of the retained block can be replaced by a block of the inverse of $\mathbf{B}^{\text {(kron) }}$ (due to Eq. (18)) it is possible do sums sequentially; see Eq. (19). Eq. (18) will be satisfied for any order of the tensor product basis functions if it is satisfied for one order. By block Gaussian elimination, [37] it is simple to prove that Eq. (18) is correct if the tensor product basis functions are ordered so that the top left block of the reordered $\mathbf{B}^{(\mathrm{kron})}$ is $\left[\mathbf{C}^{T} \mathbf{B}^{(\mathrm{kron})} \mathbf{C}\right]$ and the top right block of the reordered $\mathbf{B}^{(\mathrm{kron})}$ is zero. We have done calculations with the $\|\mathbf{i}-\mathbf{1}\| \leq b$ pruning condition and in this case it is easy to show that if the 1-D functions are ZAPPL functions then functions excluded from the tensor product basis are zero at retained points and thus Eq. (18) is satisfied.

We are now able to evaluate the sum in Eq. (8) sequentially, and when using a simple pruning condition obtain explicit equations for the upper limits of the sums. For example, for the $\|\mathbf{a}-\mathbf{1}\|_{1} \leq b$ pruning condition, one has,

$$
\begin{aligned}
C_{\mathbf{i}} & =\sum_{\|\mathbf{a}-\mathbf{1}\|_{1} \leq b}\left(\mathbf{C}^{T}\left[\mathbf{B}^{(\mathrm{kron})}\right]^{-1} \mathbf{C}\right)_{\mathbf{i}, \mathbf{a}} f\left(\mathbf{r}_{\mathbf{a}}\right) \\
& =\sum_{\|\mathbf{a}-\mathbf{1}\|_{1} \leq b}\left(\left[\mathbf{B}^{(\mathrm{kron})}\right]^{-1}\right)_{\mathbf{i}, \mathbf{a}} f\left(\mathbf{r}_{\mathbf{a}}\right) \quad \forall\|\mathbf{i}-\mathbf{1}\|_{1} \leq b \\
C_{\mathbf{i}} & =\sum_{a_{D}=1}^{a_{D}^{(\max )}}\left(\left[\mathbf{B}^{(D)}\right]^{-1}\right)_{i_{D}, a_{D}} \ldots \sum_{a_{2}=1}^{a_{2}^{(\max )}}\left(\left[\mathbf{B}^{(2)}\right]^{-1}\right)_{i_{2}, a_{2}} \sum_{a_{1}=1}^{a_{1}^{(\max )}}\left(\left[\mathbf{B}^{(1)}\right]^{-1}\right)_{i_{1}, a_{1}} f\left(\mathbf{r}_{\mathbf{a}}\right) .
\end{aligned}
$$


If the $\left[\mathbf{B}^{(k)}\right]^{-1}$ matrices were not lower triangular, then the range of possible $i_{k}$ values would be limited by

$$
i_{k}^{(\max )}=b-\sum_{k^{\prime}=1}^{k-1}\left(i_{k^{\prime}}-1\right)+1
$$

and the upper limits on the sums over $a_{k}$ would be

$$
a_{k}^{(\max )}=b-\sum_{k^{\prime}=k+1}^{D}\left(a_{k^{\prime}}-1\right)+1 .
$$

However, because $\mathbf{B}^{(k)}$ is lower triangular, $\left[\mathbf{B}^{(k)}\right]^{-1}$ is also lower triangular and therefore the upper limits of the sums are

$$
a_{k}^{(\max )}=i_{k}
$$

and

$$
i_{k}^{(\max )}=b-\sum_{k^{\prime}=1}^{k-1}\left(i_{k^{\prime}}-1\right)-\sum_{k^{\prime}=k+1}^{D}\left(a_{k^{\prime}}-1\right)+1 .
$$

Related sequential summation techniques were previously used: with a basis restricted by $\|\mathbf{j}-\mathbf{1}\|_{1} \leq b$ in Ref. [25]; for Smolyak quadrature in Refs. [6, 7]; for collocation in Ref. [2]; and for collocation with a hierarchical (ZAPPL) basis in Ref. [3, 4, 5, 30, 26]. Note that in Refs. $[6,3,4,5,30]$ a similar sequential summation idea is used with sparse grids made with either $m_{k}\left(\ell_{k}\right) \neq \ell$ or a pruning condition more general than $\|\mathbf{i}-\mathbf{1}\|_{1} \leq b$. The unidirectional principle also exploits the sequential evaluation of sums. [31, 32, 33, 36]

The numerical cost associated with the prescription in Eq. (19) can be estimated by counting the number of multiplications required for each of the $D$ sequential summations. The number of multiplications required for the sum over $a_{1}$ is

$$
\begin{aligned}
\frac{1}{D} N_{\text {mult }} & =\sum_{a_{D}=1}^{b+1} \sum_{a_{D-1}=1}^{b-\left(a_{D}-1\right)+1} \cdots \sum_{a_{2}=1}^{b-\sum_{k^{\prime}=3}^{D}\left(a_{k^{\prime}}-1\right)+1} \sum_{i_{1}=1}^{b-\sum_{k^{\prime}=2}^{D}\left(a_{k^{\prime}}-1\right)+1} \sum_{a_{1}=1}^{i_{1}} 1 \\
& =\sum_{a_{D}=0}^{b} \sum_{a_{D-1}=0}^{a_{D}} \ldots \sum_{a_{2}=0}^{a_{3}} \sum_{i_{1}=0}^{a_{2}} \sum_{a_{1}=0}^{i_{1}} 1 \\
& =\sum_{a_{D}=0}^{b} \sum_{a_{D-1}=0}^{a_{D}} \ldots \sum_{a_{2}=0}^{a_{3}} \sum_{i_{1}=0}^{a_{2}}\left(i_{1}+1\right) .
\end{aligned}
$$

The expression in Eq. (24) can be evaluated analytically [12] yielding

$$
N_{\text {mult }}=D\left(\frac{b}{D+1}+1\right) N_{\text {sparse }}
$$

which is the scaling given in Eq. (15). Eq. (15) is the cost of computing all the $C_{\mathbf{i}}$ coefficients. The transformation of Eq. (19) was used in Ref. [26, 30]. Its cost depends directly on the number of points on the sparse grid. 


\subsection{Comparison with other methods}

In the field of uncertainty quantification, $[28,29]$ it is often desirable to transform from a vector of values of a function on a sparse grid to a vector of coefficients of a so-called generalised polynomial chaos expansion (GPCE), for example, to facilitate obtaining stochastic quantities of interest. The GPCE is a special case of the sparse grid interpolation of Eq. (3), where in the GPCE case the $\varphi_{i_{k}}^{(k)}\left(x_{k}\right)$ are (weighted) polynomials. Several different methods are used to obtain the coefficients.

Some methods for computing basis expansion coefficients build a $\mathbf{B}$ matrix whose rows and columns each have a level label and a label identifying a basis function within that level. [24] Although $\varphi_{i_{k}}^{(k)}\left(x_{k}\right)$ can be chosen to make it possible to exploit the sparsity of the corresponding $\mathbf{B}$, the sums required to compute the coefficients are costly. The key advantage of our algorithm is the sequential evaluation of the sums. Algorithm 1 of Ref. [24] scales as the square of the number of functions in the basis. The scaling of Eq. (25) is much better.

Other methods for computing basis expansion coefficients manipulate separately the grids that together compose the sparse grid. An algorithm of this kind is used in Refs. [15, 13]. They solve linear systems for all the tensor product grids associated with the levels $\ell$ that satisfy $\|\boldsymbol{\ell}-\mathbf{1}\|_{1} \leq b$. For one of the tensor product grids, they write

$$
f\left(\mathbf{r}_{\tilde{\mathbf{a}}}\right)=\sum_{p_{k} \leq \ell_{k} \forall k} \beta_{\mathbf{p}}^{\ell} L_{\mathbf{p}}^{\ell}\left(\mathbf{r}_{\tilde{\mathbf{a}}}\right)
$$

where $L_{\mathbf{p}}^{\ell}$ is a basis function for the tensor product grid labelled by $\ell$, and solve for $\beta_{\mathbf{p}}^{\ell}$ for each tensor product grid separately. Coefficients with the same $\mathbf{p}$ for different grids must be combined to determine $C_{\mathbf{i}} \cdot \mathbf{r}_{\tilde{a}}$ is a point on the tensor product grid associated with $\boldsymbol{\ell}$; the union of the grids whose points are denoted $\mathbf{r}_{\tilde{\mathbf{a}}}$ is the sparse grid on which the points are denoted by $\mathbf{r a}_{\mathbf{a}}$.

The total cost of this separate grids method is the sum of the costs of matrix-vector products for the grids, $N_{\text {mult, } 2}^{(\mathrm{sep})}$, and the cost of inverting matrices for the grids, $N_{\text {mult }, 3}^{(\mathrm{sep})}$,

$$
\text { cost }=N_{\text {mult }, 2}^{(\text {sep }}+N_{\text {mult }, 3}^{(\text {sep }}
$$

where

$$
N_{\mathrm{mult}, n}^{(\mathrm{sep})}=\sum_{\|\ell-\mathbf{1}\|_{1} \leq b} \prod_{k=1}^{D} \ell_{k}^{n} .
$$

In this equation, we assume that the number of points for coordinate $x_{k}$ in level $\ell_{k}$ is equal to $\ell_{k}$ and that the cost of the matrix-vector product required to obtain $\beta_{\mathbf{p}}^{\ell}$ is $\left(\prod_{k=1}^{D} \ell_{k}\right)^{2}$. If the matrix whose elements are $L_{\mathbf{p}}^{\ell}\left(\mathbf{r}_{\tilde{\mathbf{a}}}\right)$ is orthogonal, and there is therefore no need to invert it, $N_{\text {mult, } 2}^{(\text {sep })}$ is the total cost. On the other hand, when $L_{\mathbf{p}}^{\ell}\left(\mathbf{r}_{\tilde{\mathbf{a}}}\right)$ is not orthogonal, then $N_{\text {mult, } 3}^{(\text {sep })}$ must be included in Eq. (27). It is also possible to exploit the tensor product character of the grids.

After some algebraic transformations, Eq. (28) can be cast into a form similar to Eq. (24). However, it does not seem to be possible to find an equation in closed form for $N_{\text {mult }, n}^{(\text {sep }}$ for a general $D$. [12] We thus evaluate Eq. (28) numerically, to compare the cost of this separategrids method and the approach in Sect. 3.2. We plot the numbers of operations, in Eq. (25) and Eq. (28), as a function of $D$ for the threshold parameters $b=4, b=9$, and $b=14$ in Figs. 1, 2, and 3, respectively. For Figure 1, Figure 2, and Figure 3, the columns that are deleted are those for which $\|\mathbf{i}-\mathbf{1}\|_{1}>b$. 
Fig. 1: Scaling of floating point operations as a function of $D, b=4$ (i.e. 5 points per coordinate)

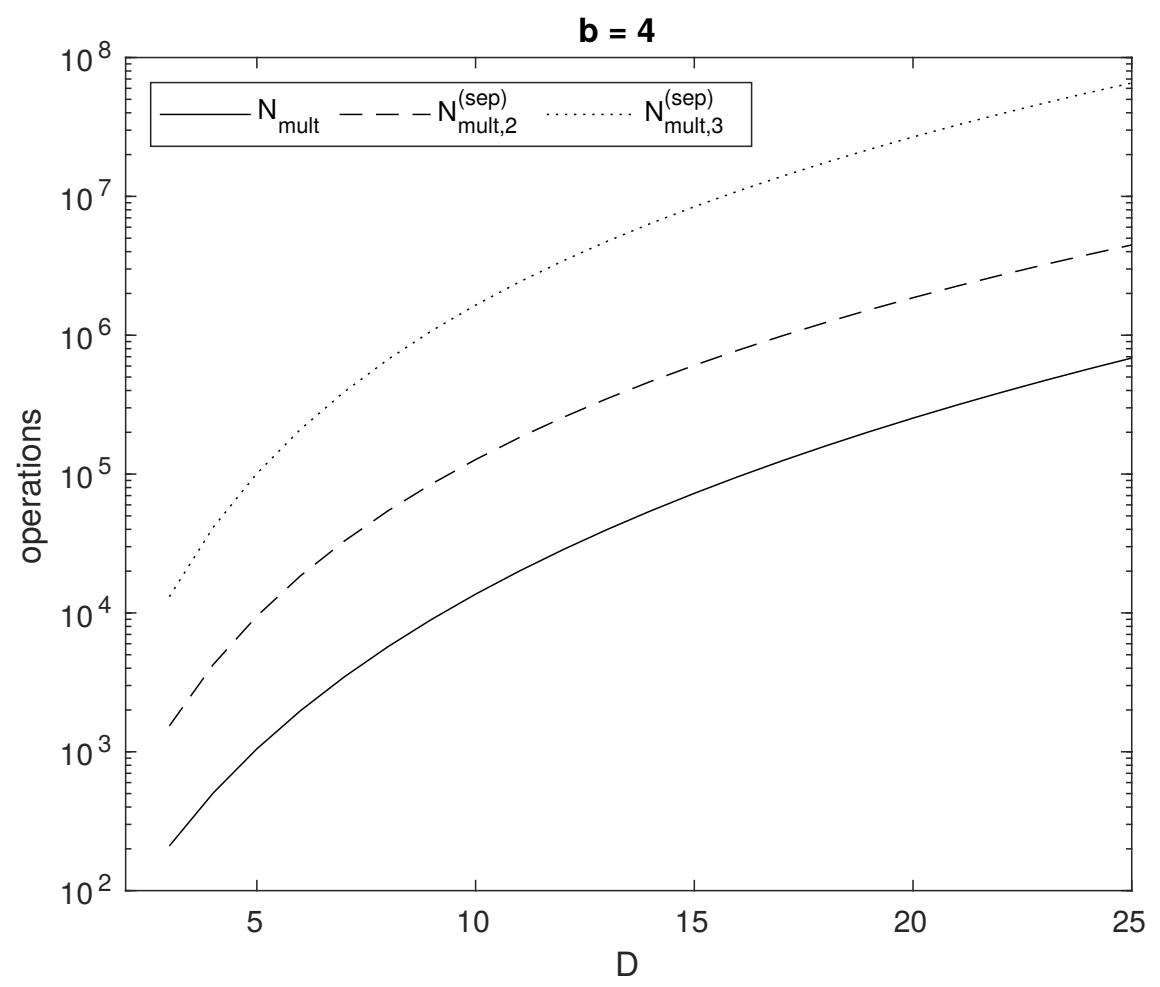


Fig. 2: Scaling of floating point operations as a function of $D, b=9$ (i.e. 10 points per coordinate)

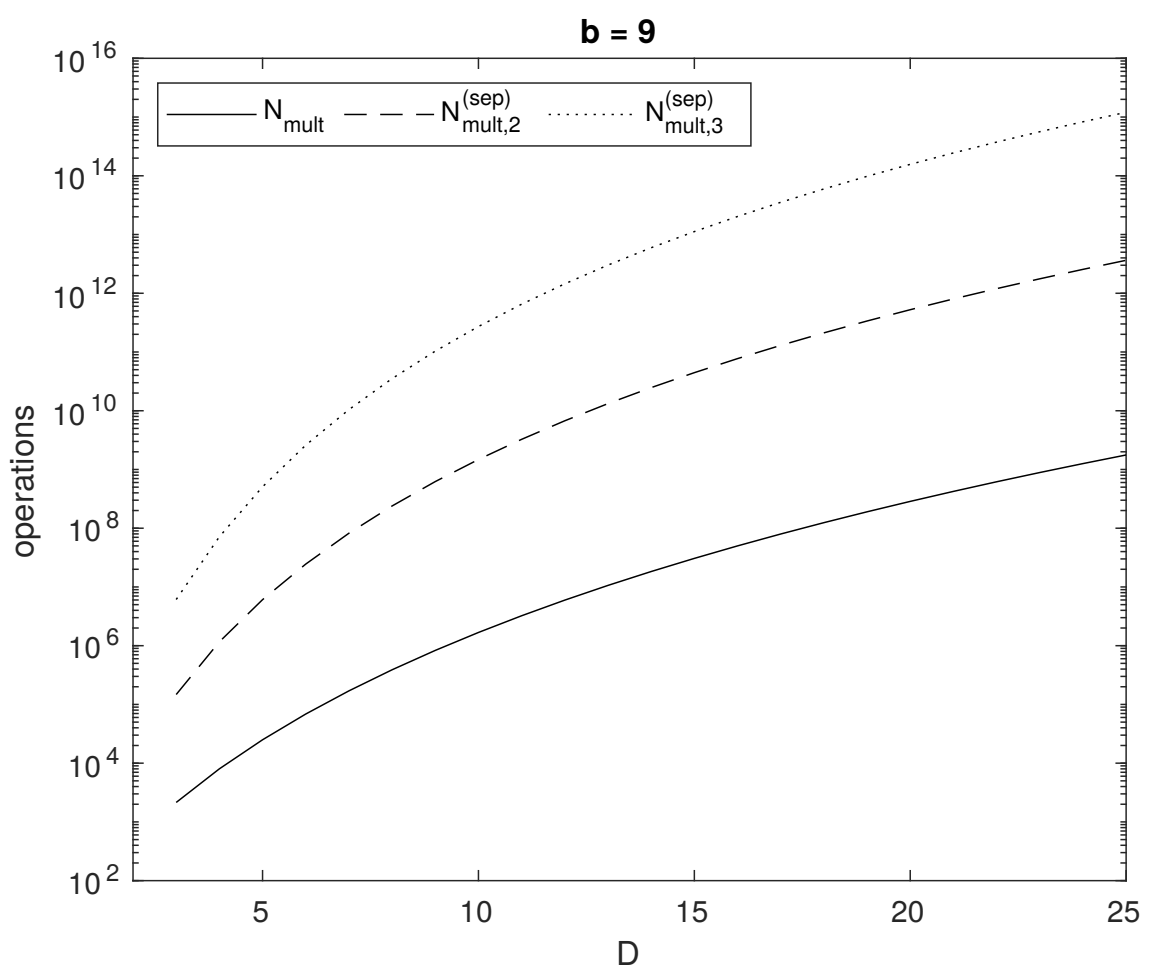


Fig. 3: Scaling of floating point operations as a function of $D, b=14$ (i.e. 15 points per coordinate)

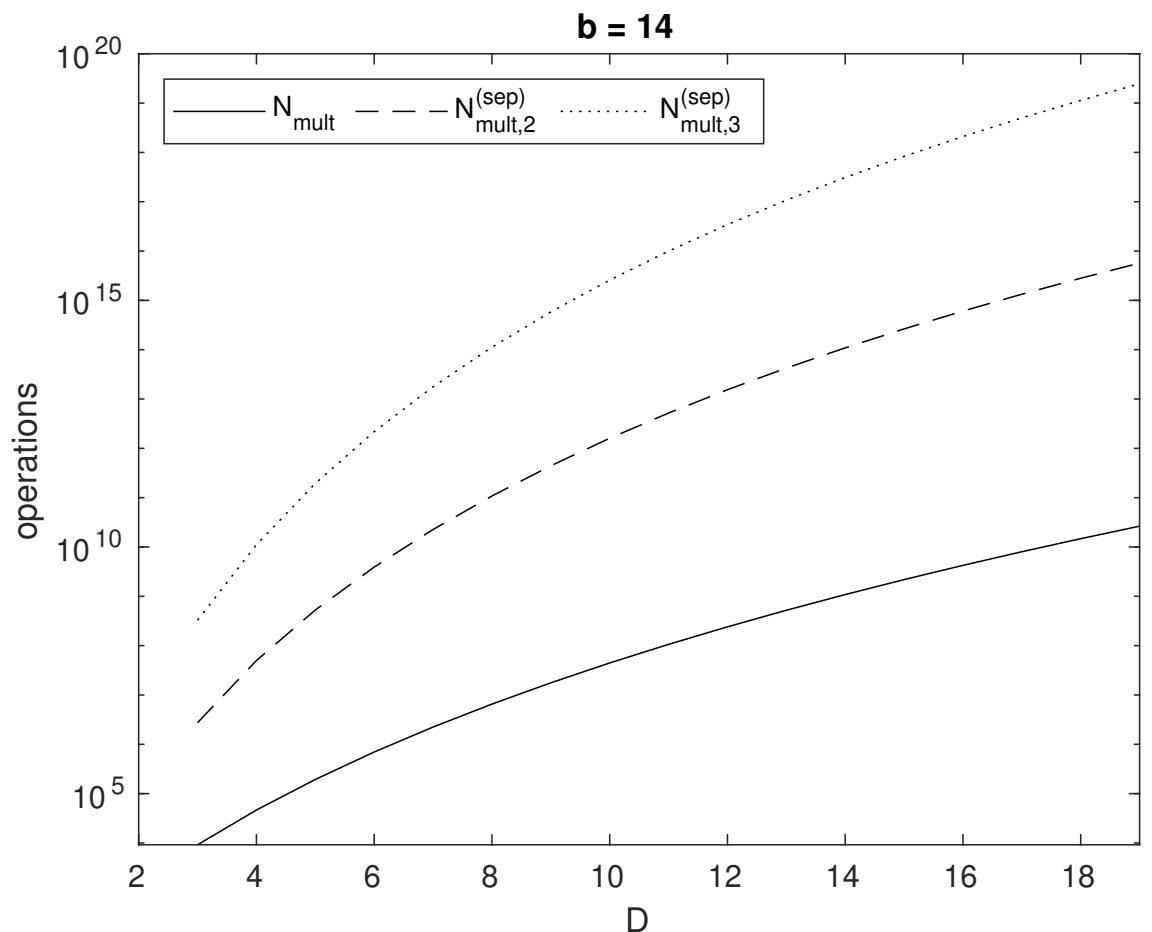

When $b=9$ and $b=14$ the sequential summation method of Sect. 3.2 is orders of magnitude less costly than the separable grids method.

\section{Conclusion}

In this chapter, we explain that by doing sums sequentially it is possible to efficiently obtain expansion coefficients in a basis of products of 1-D global functions from values of a function on a sparse grid. Such coefficients are needed in many contexts. They are needed whenever one wishes to make an interpolant from a sparse grid and global 1-D functions. For example, in Sect. 2.6 of Ref. [14] the coefficients, called polynomial chaos expansion coefficients, are determined by evaluating integrals with Smolyak quadratures. In Sect. 2.7 of Ref. [14], the coefficients are obtained by solving a system of linear equations whose size is equal to the number of sparse grid points. If both the basis and the grid are tensor products, it is well established that basis coefficients can be computed from function values by doing sums sequentially. See for example, Table 10.1 of Ref. [10]. By doing sums sequentially, one obviates the need to loop simultaneously over all the indices. It is not well established that a similar sequential summation idea can be used when the grid and the basis are built from a general sparse grid recipe and nested sets of 1-D grids and global 1-D bases. In Ref. [3], the original sparse grid 
interpolation, in terms of products of differences of 1-D interpolation operators, was formulated using sequential sums. In this chapter, we have shown that it is also possible to interpolate, i.e., to compute basis expansion coefficients, using sequential sums, without writing the interpolant in terms of products of differences of 1-D interpolants. The sequential summation approach is considerably less costly than established methods.

\section{Relation with the Chapter of David Holzmueller and Dirk Pflueger}

We have read a preliminary version of the chapter by Holzmueller and Pflueger and wish to clarify the relationship between their contribution and ours. First, in many of our equations we use a special and simple restriction to determine which functions (points) are included in the sparse basis (grid): $\|\mathbf{i}-\mathbf{1}\| \leq b$. This enables to write out the sums in Eq. (19) explicitly and to impose the restriction by using the appropriate upper limits on the sums. Holzmueller and Pflueger do not explain in detail how they evaluate matrix vector products, but appear to use index lists and their formalism allows one to use much more general restrictions. Ideas similar to those in this paper can be used with the restriction $g_{1}\left(i_{1}-1\right)+g_{2}\left(i_{2}-1\right)+\cdots+g_{D}\left(i_{D}-1\right) \leq b$, where $g_{c}\left(i_{c}-1\right), c=1, \cdots D$, is a monotonically increasing function.[3, 30]. In this case it is also possible to derive equations for the upper limits on the sums in Eq. (19). Much more general restrictions can be used if one is willing to forgo deriving equations for the upper limits and instead uses index lists. [34, 35] Second, in this paper we have one function (point) per level. Holzmueller and Pflueger have no such constraint. However, this restriction can be lifted.[3] Third, we have not given a recipe for choosing 1-D basis functions and dividing the tensor product functions obtained from them into two groups, labelled "retained" and "discarded", so that all the discarded functions are zero at the retained points. This is simple if the 1-D functions are ZAPPL functions and all the functions that satisfy $g_{1}\left(i_{1}-1\right)+g_{2}\left(i_{2}-1\right)+\cdots+$ $g_{D}\left(i_{D}-1\right) \leq b$ are retained $\left(g_{c}\left(i_{c}-1\right)\right.$ is a monotonically increasing function). It is in general not simple if some of the functions that satisfy $g_{1}\left(i_{1}-1\right)+g_{2}\left(i_{2}-1\right)+\cdots+g_{D}\left(i_{D}-1\right) \leq b$ are excluded, i.e., if there are holes. An easy, and often inexpensive, way to avoid holes is to plug them by adding functions to the retained basis. When using spatially localised basis functions, it is sometimes advantageous to discard functions in regions in which the function being interpolated is smooth, i.e. to introduce holes. In the sparse grid literature this is known as spatial adaptivity. When using global basis functions, often best for representing a smooth function, spatial adaptivity is less useful.

\section{Acknowledgements}

Research reported in this article was funded by The Natural Sciences and Engineering Research Council of Canada. We thank David Holzmueller for sending us reference [13], for emails about his LU perspective. We are grateful to both David Holzmueller and Dirk Pflueger for discussions. 


\section{References}

1. The quadrature of the parabola. http://en.wikipedia.org/wiki/The_ Quadrature_of_the_Parabola.

2. Gustavo Avila and Tucker Carrington, Solving the Schroedinger equation using Smolyak interpolants. J. Chem. Phys. 139, 134114 (2013)

3. Gustavo Avila and Tucker Carrington, A multi-dimensional Smolyak collocation method in curvilinear coordinates for computing vibrational spectra. J. Chem. Phys. 143, 214108 (2015)

4. Gustavo Avila and Tucker Carrington, Computing vibrational energy levels of $\mathrm{CH}_{4}$ with a Smolyak collocation method. J. Chem. Phys. 147, 144102 (2017)

5. Gustavo Avila and Tucker Carrington, Reducing the cost of using collocation to compute vibrational energy levels: results for $\mathrm{CH}_{2} \mathrm{NH}$. J. Chem. Phys. 147, 064103 (2017)

6. Gustavo Avila and Tucker Carrington, Nonproduct quadrature grids for solving the vibrational Schroedinger equation. J. Chem. Phys. 131, 174103 (2009)

7. Gustavo Avila and Tucker Carrington, Using a pruned basis, a non-product quadrature grid, and the exact Watson normal-coordinate kinetic energy operator to solve the vibrational Schroedinger equation for $\mathrm{C}_{2} \mathrm{H}_{4}$. J. Chem. Phys. 135, 064101 (2011)

8. Gustavo Avila, Jens Oettershagen, and Tucker Carrington, Comparing nested sequences of Leja and Pseudogauss points to interpolate in 1-D and solve the Schroedinger equation in 9-D. In: Sparse Grids and Applications (Springer International Publishing, Miami, 2016), pp. 1-17

9. Volker Barthelmann, Erich Novak, and Klaus Ritter, High dimensional polynomial interpolation on sparse grids. Adv. Comput. Math. 12, 273 (2000)

10. John P. Boyd, Chebyshev \& Fourier Spectral Methods, 2nd edn. (Dover Publications, New York, 2001)

11. H.-J. Bungartz and M. Griebel, Sparse grids. Acta Numerica 13, 147 (2004)

12. S. Butler and P. Karasik, A note on nested sums. J. of Int. Seq. 13, 1 (2010)

13. G. T. Buzzard, Efficient basis change for sparse-grid interpolating polynomials with application to t-cell sensitivity analysis. Comput. Bio. J. 2013, 1 (2013)

14. Michael S. Eldred, S. L. Brown, Brian M. Adams, Daniel M. Dunlavy, David M. Gay, Laura Painton Swiler, William E. Hart, J. D. Griffin, P. D. Hough, Tammy G. Kolda, and Monica Martinez-Canales, Dakota , a multilevel parallel object-oriented framework for design optimization, parameter estimation, uncertainty quantification, and sensitivity analysis version 4.0 user's manual. (2002) https: / / dakota. sandia.gov/content/ manuals

15. L. Formaggia, A. Guadagnini, I. Imperiali, V. Lever, G. Porta, M. Riva, A. Scotti, and L. Tamellini, Global sensitivity analysis through polynomial chaos expansion of a basinscale geochemical compaction model. Comput. Geosci. 17, 25 (2013)

16. Klaus Hallatschek, Fouriertransformation auf dünnen Gittern mit hierarchischen Basen. Numerische Mathematik 63, 83 (1992)

17. D. Holzmueller, private communication. (2018)

18. Andreas Klimke and Barbara Wohlmuth, Algorithm 847: Spinterp: Piecewise multilinear hierarchical sparse grid interpolation in matlab. ACM Trans. Math. Softw. 31, 561 (2005)

19. F. Leja, Sur certaines suites liées aux ensembles plans et leur application à la représentation conforme. Ann. Polonici Math. 4, 8 (1957)

20. J. C. Light and Tucker Carrington, Discrete variable representations and their utilization. Adv. Chem. Phys. 114, 263 (2000)

21. G. Mastroianni and D. Occorsio, Lagrange interpolation at Laguerre zeros in some weighted uniform spaces. Acta Math. Hung. 91, 27 (2001) 
22. A. Narayan and J. D. Jakeman, Adaptive Leja sparse grid construction for stochastic collocation and high-dimensional approximation. SIAM J. Sci. Comput. 36, 2952 (2014)

23. Dirk Pflüger, Spatially Adaptive Sparse Grids for High-Dimensional Problems (Verlag Dr. Hut, München, 2010)

24. Julian Valentin and Dirk Pflüger, Fundamental splines on sparse grids and their application to gradient-based optimization. In: Sparse Grids and Applications (Springer International Publishing, Miami, 2016), pp. 229-251

25. X.-G. Wang and Tucker Carrington, The utility of constraining basis function indices when using the Lanczos algorithm to calculate vibrational energy levels. J. Phys. Chem. A 105, 2575 (2001)

26. Robert Wodraszka and Tucker Carrington, A pruned collocation-based multi-configuration time-dependent Hartree approach using a Smolyak grid for solving the Schrödinger equation with a general potential energy surface. J. Chem. Phys. 150, 154108 (2019)

27. D. Xiu and G. Karniadakis, The Wiener-Askey polynomial chaos for stochastic differential equations. SIAM Journal on Scientific Computing 24, 619 (2002)

28. Ivo Babuška, Fabio Nobile, Raúl Tempone, A Stochastic Collocation Method for Elliptic Partial Differential Equations with Random Input Data, SIAM REVIEW Vol. 52, No. 2, pp. 317-355, (2010)

29. D. Xiu and J. S. Hesthaven, High-order collocation methods for differential equations with random inputs, SIAM J. Sci. Comput., 27 1118-1139 (2005)

30. Emil J. Zak and Tucker Carrington, Using collocation and a hierarchical basis to solve the vibrational Schrödinger equation J. Chem. Phys. 150, 204108 (2019)

31. R. Balder, Adaptive Verfahren für elliptische und parabolische Differentialgleichungen auf dünnen Gittern. Dissertation, Technische Universität Dissertation, Technische Universität München, 1994

32. C. Feuersaenger, Sparse Grid Methods for Higher Dimensional Approximation. Dissertation, Institut für Numerische Simulation, Universität Bonn, 2010

33. D. Pflueger, Spatially adaptive sparse grids for high-dimensional problems. Dissertation, Technische Unversität München, 2010

34. R. Wodraszka and T. Carrington, Using a pruned, nondirect product basis in conjunction with the multi-configuration time-dependent Hartree (MCTDH) method J. Chem. Phys. 145, 044110 (2016)

35. R. Wodraszka and T. Carrington, Systematically expanding nondirect product bases within the pruned MCTDH method: A comparison with multi-layer MCTDH J. Chem. Phys. 146, 194105 (2017)

36. A. Zeiser, Fast matrix-vector multiplication in the sparse-grid Galerkin method J. Sci. Comput. 47, 328 (2011)

37. Tzon-Tzer Lu and Sheng-Hua Shiou, Computers and Mathematics with Applications 43, $119-129$ (2002) 